\title{
Multifrequency STD NMR Unveils the Interactions of Antibiotics With Burkholderia multivorans Biofilm Exopolysaccharide
}

\author{
Ridvan Nepravishta ${ }^{1}$, Serena Monaco ${ }^{1}$, Marco Distefano ${ }^{2}$, Roberto Rizzo ${ }^{2}$, Paola Cescutti ${ }^{2}$ \\ and Jesus Angulo ${ }^{1,3,4 *}$
}

${ }^{1}$ School of Pharmacy, University of East Anglia, Norwich, United Kingdom, ${ }^{2}$ Department Life Sciences, University of Trieste, Trieste, Italy, ${ }^{3}$ Department of Organic Chemistry, Faculty of Chemistry, University of Seville, Seville, Spain, ${ }^{4}$ Instituto de Investigaciones Químicas (CSIC-US), Seville, Spain

OPEN ACCESS

Edited by:

Jesus Jimenez-Barbero,

CIC bioGUNE, Spain

Reviewed by:

Filipa Marcelo,

New University of Lisbon, Portugal Alba Silipo,

University of Naples Federico II, Italy

*Correspondence: Jesus Angulo jangulo@us.es

Specialty section: This article was submitted to Structural Biology,

a section of the journal

Frontiers in Molecular Biosciences

Received: 20 June 2021

Accepted: 02 August 2021 Published: 16 September 2021

Citation:

Nepravishta R, Monaco S, Distefano M, Rizzo R, Cescutti $P$ and Angulo J (2021) Multifrequency STD

NMR Unveils the Interactions of Antibiotics With Burkholderia multivorans

Biofilm Exopolysaccharide. Front. Mol. Biosci. 8:727980. doi: 10.3389/fmolb.2021.727980
Biofilms confine bacterial cells within self-produced matrices, offering advantages such as protection from antibiotics and entrapment of nutrients. Polysaccharides are major components in these macromolecular assemblies, and their interactions with other chemicals are of high relevance for the benefits provided by the biofilm 3D molecular matrix. NMR is a powerful technique for the study and characterization of the interactions between molecules of biological relevance. In this study, we have applied multifrequency saturation transfer difference (STD) NMR and DOSY NMR approaches to elucidate the interactions between the exopolysaccharide produced by Burkholderia multivorans C1576 (EpolC1576) and the antibiotics kanamycin and ceftadizime. The NMR strategies presented here allowed for an extensive characterization at an atomic level of the mechanisms behind the implication of the EpolC1576 in the recalcitrance phenomena, which is the ability of bacteria in biofilms to survive in the presence of antibiotics. Our results suggest an active role for EpolC1576 in the recalcitrance mechanisms toward kanamycin and ceftadizime, though through two different mechanisms.

Keywords: STD NMR, multifrequency STD NMR, exopolysaccharides, biofilms, Burkholderia multivorans

\section{INTRODUCTION}

In all living organisms, several strategies have been developed with regard to intercellular communication and mechanical or xenobiotic defense. At the molecular level, carbohydrate-carbohydrate or carbohydrate-protein interactions are essential parts of these biological processes. Specifically, in bacterial infections, these interactions are of paramount importance in the early stages of cell-host adhesion or in the bacterial collectivity life modality called biofilm (BF) (Flemming et al., 2016). With regard to the latter, a relevant way of bacterial colony life is their growth in self-produced matrices having a gel-like structure typical of the BF lifestyle. The composition of the matrices is chemically heterogeneous, including proteins, exopolysaccharides (Epols), and DNA, with Epols as the most abundant part of the BF and important for the matrix's mechanical stability. The chemical structure of BF's Epols is known only for a few bacterial species; however, depending on the type of Epol produced by the bacteria, it is presumed that either polar or hydrophobic non-covalent interactions might be, in general, involved 
in the resulting 3D assembly (Flemming, 2016). The internal architecture of Epols and their detailed mechanisms of interactions conferring mechanical stability to the biofilm still remain, up to date, elusive.

BF matrices take part actively in interactions with molecules of the surrounding environment (Limoli et al., 2015), and in particular, Epols have been shown to bind small molecules (Worthington et al., 2012), suggesting a possible active participation of Epols in many processes positively influencing bacterial life. Globally, the molecular interactions between Epols and small molecules constitute a network of molecular recognition processes that have great importance for the colony, mainly regarding nutrition, defense, and cell-cell communication. In fact, considering the nutrition aspect, the interactions of nutrients with the BF lead to a slow and constant diffusion of these molecules toward the colony. With regard to defense, recalcitrance, a multifactorial reversible defense phenomenon, confers non-susceptibility to bacteria toward antibiotics that disappear when the $\mathrm{BF}$ is disrupted. As with the nutrients, the interactions of antibiotics with the BF significantly impact their effectiveness, and a corresponding reduction in penetration to the cells has been demonstrated to be one of the mechanisms contributing to recalcitrance (Hall and Mah, 2017).

In this study, we wanted to unveil the molecular basis for some of the interactions that underlie the involvement of Epols in the defense mechanisms of bacteria, such as recalcitrance, demonstrating that the polysaccharide extracted from $\mathrm{BF}$ produced by Burkholderia multivorans reference strain C1576 (hereafter EpolC1576) interacts with ceftazidime (a cephalosporin antibiotic) and kanamycin (an aminoglycoside antibiotic) by two completely different molecular mechanisms. B. multivorans is part of the so-called Burkholderia cepacia complex, a family of bacteria; many of them involved in serious lung infections in cystic fibrosis patients that might evolve in premature death following severe decline in the lung function (Scoffone et al., 2017). B. multivorans on Mueller-Hinton agar produces a rhamno-mannan Epol containing $\alpha$-D-rhamnose and $\alpha$-D-mannose residues in equimolar ratios, with $50 \% \mathrm{O}$-methyl substitution on C-3 of the 2-linked rhamnose residues (Scheme 1) (Dolfi et al., 2015).

Accessing the possible mechanisms that can contribute to recalcitrance at the molecular level is not trivial. Very few biophysical techniques can achieve a depth in structural information at an atomic level without losing the focus on the dynamical interactions between the small molecule and the exopolysaccharide. Here, we hypothesized that the ${ }^{1} \mathrm{H}$ saturation transfer difference (STD) NMR spectroscopy (Mayer and Meyer, 1999) could provide molecular level details of the interactions of BF-forming exopolysaccharides and small molecules. ${ }^{1} \mathrm{H}$ STD NMR is the technique of choice for studies typically focusing on the interactions between a small ligand and a protein. Relying on a fast exchange kinetics regime between the free and bound states of the ligand, it gives powerful insights about the binding epitope (Mayer and James, 2004) and dissociation constant $\left(\mathrm{K}_{\mathrm{D}}\right)$ (Angulo et al., 2010), and, as lately shown, also about ligand orientation in the binding site (Monaco et al., 2017). Inspirational to our experimental setup in this study was the application of the selective frequency saturation transfer difference technique applied to the binding of small molecules to DNA (Di Micco et al., 2005; Martin et al., 2005; Souard et al., 2008). Differently to other NMR techniques, the strength of STD NMR is based on the fact that there is virtually no limitation on the upper limit of the molecular weight of the receptor. Based on this strength, many groups have explored the use of STD NMR to characterize the interaction of small molecules with viruses (Benie et al., 2003), receptors embedded at the outer region of the eukaryotic cell (Airoldi et al., 2011), and, lately, also interaction of small molecules with soft mater, such as hydrogels (Calabrese et al., 2019).

\section{MATERIALS AND METHODS}

\section{Bacterial Strain, Media, and Chemicals}

B. multivorans C1576 (LMG 16660) is a reference strain that belongs to the panel of $B$. cepacia complex strains (EP1), and it was purchased from the BCCMTM bacteria collection. Mueller-Hinton Broth (MHB) and Bacto Agar were purchased from Difco Laboratories. The fluorescent probes 1-anilino-8naphthalene-sulfonate sodium salt ( $\mathrm{Na}+$ ANS-) and 2(p-toluidinyl)-6-naphthalene sulfonate potassium salt (K + TNS-) as well as antibiotics kanamycin sulfate salt (Kanamycin A) and ceftazidime were purchased from SigmaAldrich.

Biofilms of B. multivorans $\mathrm{C} 1576$ were developed on cellulose membranes (Sigma, cutoff $12.400 \mathrm{Da}$ ) treated as follows: membranes were cut in circles to fit Petri dishes, boiled in $5 \% \mathrm{Na} 2 \mathrm{CO} 3$ solution for $15 \mathrm{~min}$ and then boiled in water for $15 \mathrm{~min}$, subjected to autoclave sterilization, and laid on Petri dishes containing the $\mathrm{MH}$ agar medium. Membranes were extended all over the plate, and surplus water was let to evaporate under the hood. An overnight liquid culture of $B$. multivorans C1576 was diluted to obtain a cell suspension having $0.13 \mathrm{OD}$ at $600 \mathrm{~nm}$, and three aliquots of $10 \mu \mathrm{l}$ of the cell dilution were transferred on the membranes. The same liquid medium was used for the overnight culture of $B$.

\section{[2)- $\alpha$-D-Manp-( $1 \rightarrow 2)-\alpha$-D-Rhap-(1 $\rightarrow 3)-\alpha-D-R h a p-(1 \rightarrow 3)-\alpha-D-M a n p-(1 \rightarrow]_{\mathrm{n}}$

SCHEME 1 | Repeating unit of the exopolysaccharide produced by B. multivorans C1576 (EpolC1576). 
multivorans $\mathrm{C} 1576$ and for Petri dish filling. After 7 days of incubation at $30^{\circ} \mathrm{C}$, the biofilm on the membranes was recovered with sterile $0.9 \% \mathrm{NaCl}$, and then the cell suspension was added with $\mathrm{NaOH}$ to reach a final concentration of $0.1 \mathrm{M}$ and incubated at room temperature, with shaking for $3 \mathrm{~h}$ to dismantle the biofilm. The cell suspension was then centrifuged at $40,000 \mathrm{~g}$ at $4^{\circ} \mathrm{C}$ for $30 \mathrm{~min}$, and the supernatant, containing soluble EpolC1576, was recovered and dialyzed against distilled water using a $12-14 \mathrm{kDa}$ molecular weight cutoff (MWCO, SERVA Electrophoresis $\mathrm{GmbH}$, Heidelberg, Germany) membrane for $72 \mathrm{~h}$ at $25^{\circ} \mathrm{C}$. The solution recovered from a dialysis tube was cooled in ice and added with trichloroacetic acid $(20 \% \mathrm{w} / \mathrm{v}$ final concentration) to precipitate proteins and nucleic acids. After $30 \mathrm{~min}$ of incubation, the solution was centrifuged $40,000 \mathrm{~g}$ for $30 \mathrm{~min}$ at $4{ }^{\circ} \mathrm{C}$, and the supernatant was collected and dialyzed against distilled water using a $12-14 \mathrm{kDa}$ MWCO membrane for $72 \mathrm{~h}$ at $25^{\circ} \mathrm{C}$. The dialysate was cooled in ice and added with 4 volumes of $96 \%$ cold ethanol, and the mixture was placed for $24 \mathrm{~h}$ at $-20^{\circ} \mathrm{C}$ to separate the polysaccharide from lipids. The solution was then centrifuged at $40,000 \mathrm{~g}$ for $30 \mathrm{~min}$ at $4^{\circ} \mathrm{C}$, and the pellet consisting of polysaccharide was resuspended in Milli$\mathrm{Q}$ water and dialyzed against the same for $72 \mathrm{~h}$ at $4^{\circ} \mathrm{C}$ using a $12-14 \mathrm{kDa}$ MWCO membrane. The remaining retentate was taken to neutral $\mathrm{pH}$, filtered through a $0.22-\mu \mathrm{m}$ pore size filter (KX Sterile Syringe Filter, Kinesis), and lyophilized. The purity of the EpolC1576 sample used in STD NMR experiments was assessed by means of ${ }^{1} \mathrm{H}$ NMR, which also confirmed the primary structure (Dolfi et al., 2015, 411, 42-48.). The full ${ }^{1} \mathrm{H}$ NMR spectrum is showed in Supplementary Figure S1; the integration values of anomeric proton peaks and rhamnose methyl group are in perfect agreement with the structure. No signals other than those pertaining to EpolC1576 are present in the spectrum, thus indicating that the sample was suitable to STD experiments. EpolC1576 was exchanged three times with $99.9 \% \quad \mathrm{D}_{2} \mathrm{O}$ (Sigma-Aldrich) by lyophilization and finally dissolved in $0.6 \mathrm{ml}$ of $99.9 \% \mathrm{D}_{2} \mathrm{O}$ before recording a ${ }^{1} \mathrm{H}$ NMR spectrum on a $500-\mathrm{MHz}$ Varian spectrometer operating at $50^{\circ} \mathrm{C}$.

\section{STD NMR Experiments}

All the NMR experiments were conducted using a Bruker Avance II $500 \mathrm{MHz}$ machine in $20 \mathrm{mM}$ Tris-d11 $\mathrm{D}_{2} \mathrm{O}$ buffer $\mathrm{pH} 7.5$ at 298 K. 1D ${ }^{1}$ H STD NMR spectra (Mayer and Meyer, 1999) were acquired with SW of $16 \mathrm{ppm}$ using a TD of $32 \mathrm{~K}$ data points using a recycling delay (D1) of $6 \mathrm{~s}$ with 64 scans for epitope mapping calculation. The Bruker library pulse stdiffesgp. 3 was used for all the STD NMR experiments applying a train of 50-ms Gaussianshaped pulse at $40 \mathrm{ppm}$ (off-resonance spectra) and 3.2, $-1.0 \mathrm{ppm}$ for ANS while 1.22 and -1.0 ppm for kanamycin (on-resonance spectra). The residual water signal was suppressed by the excitation sculpting technique, while the Epol signals where possible were canceled using a $40-\mathrm{ms}$ T1 $\rho$ filter. In order to obtain the epitope mappings for average orientation of the small molecules, the saturation time was varied from 0.5 to $6 \mathrm{~s}$ for each chosen saturation frequency. The results obtained were fitted using the equation $\operatorname{STD}_{(\text {tsat })}=\operatorname{STD}_{\max }\left(1-\exp \left(\mathrm{k}_{\text {sat }}{ }^{\star} \mathrm{t}_{\text {sat }}\right)\right)$, where
$\mathrm{STD}_{\text {(tsat) }}=\mathrm{I}_{0}-\mathrm{I} / \mathrm{I}_{0}$. Here, "I $\mathrm{I}_{0}$ " is the intensity of the chosen peak measured from "off-resonance" spectra, while "I" is the intensity of the same peak from the so-called "on-resonance" spectra. $\mathrm{STD}_{0}$ can be obtained easily using the equation $\mathrm{STD}_{0}=\mathrm{k}_{\mathrm{sat}} * \mathrm{STD}_{\max }$. The data were then normalized to the highest $\mathrm{STD}_{0}$ in order to obtain $\mathrm{STD}_{0}(\%)$. The $\mathrm{STD}_{0}(\%)$ factor was classified as strong for values between $81-100 \%$, as intermediate for values between $50-80 \%$, and weak for values obtained between $0-49 \%$. This scale assures the analysis of proximity, on average, of the ligand atom to the interface with the EpolC1576 and is derived for each saturation frequency.

The DOSY experiment with the aim to characterize the products of degradation was performed using the Bruker library pulse sequence ledbpgppr2s (Wu et al., 1995). The SW for the experiment was set to $16 \mathrm{ppm}$, the big delta $(\Delta)$ was set to $100 \mathrm{~ms}$, the little delta $(\delta)$ was set to $3 \mathrm{~ms}$, while D1 was set to $2 \mathrm{~s}$. A linear gradient spanning from 5 to $95 \%$ in 16 points was used. The data were fitted using the T1/T2 module and transformed in 2D DOSY spectra using the DOSY routine for topspin 3.6.

\section{Sample Preparation}

The interaction experiments between ANS and EpolC1576 were conducted at a concentration of $5 \mathrm{mM}$ and $0.5 \mathrm{mg} / \mathrm{ml}$. Not knowing the exact MW of EpolC1576, the ratio was chosen empirically as the best ratio to obtain a good STD NMR signal while attempting to maintain the EpolC1576 concentration as low as possible. For the interaction and competition studies between ANS and kanamycin or ceftazidime, the same concentrations as for interaction experiments were used for EpolC1576 and ANS $(0.5 \mathrm{mg} / \mathrm{ml}$ and $5 \mathrm{mM})$, while the concentration of kanamycin or ceftazidime was changed from 0.5 to $2 \mathrm{mM}$. The change in the intensity of the STD NMR for ANS peaks was monitored accordingly (see the text). Degradation studies with the aim to focus on the small amounts of the degradation product (pyridine) required higher amount of ceftazidime that in this case was used at a concentration of $6 \mathrm{mM}$, while the EpolC1576 was kept at the usual concentration of $0.5 \mathrm{mg} / \mathrm{ml}$. All the described experiments were performed in $20 \mathrm{mM}$ Tris-d11 $\mathrm{D}_{2} \mathrm{O}$ buffer $\mathrm{pH} 7.5$ at $298 \mathrm{~K}$. All control STD NMR experiments in order to access direct saturation for ANS, ceftazidime, and kanamycin were performed at the concentration of 5,6 , and $2 \mathrm{mM}$, respectively, in the absence of EpolC1576 in $20 \mathrm{mM}$ Tris-d11 $\mathrm{D}_{2} \mathrm{O}$ buffer $\mathrm{pH} 7.5$ at $298 \mathrm{~K}$. No direct saturation was observed.

\section{RESULTS AND DISCUSSION}

\section{STD NMR Is a Sensitive Technique to Detect and Characterize the Interactions of the Exopolysaccharide With Small Molecules}

We first wanted to probe the feasibility of the STD NMR approach for this type of interaction. The hydrophobic properties of EpolC1576 have been previously studied through its interactions with small molecules as 2-(p-toluidinyl)- 

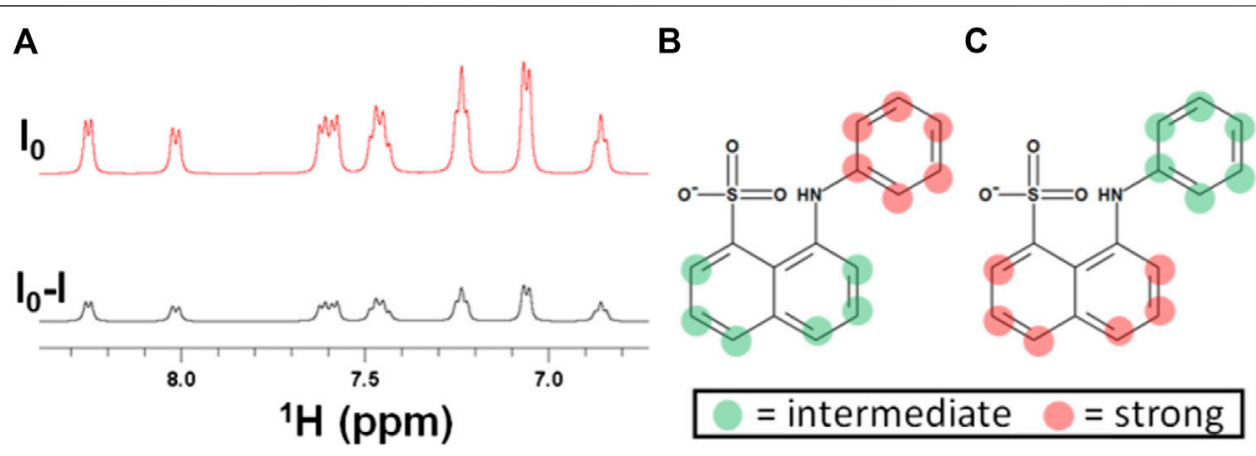

FIGURE 1 | Detection and differential-binding epitope characterization of the interaction of ANS with EpolC1576. (A) STD NMR spectra of ANS (5 mM) in the presence of EpolC1576. (B) ANS-binding epitope obtained by selectively saturating EpolC1576 at +1.22 ppm and (C) by selectively saturating EpolC1576 at +3.22 ppm. A comparison of $\mathbf{( B )}$ and $(\mathbf{C})$ indicates that the phenyl ring of ANS orients, on average, toward the $\mathrm{CH}_{3}$ moiety of EpolC1576. Intermediate STD $(\%)=$ $50-80 \%$ and strong STD $_{0}(\%)=80-100 \%$.

naphthalene-6-sulfonate (TNS) and 1-anilinonaphthalene-8sulfonate (ANS) (Kuttel et al., 2017). In that study, interactions of both compounds with EpolC1576 were demonstrated by simple observation of line broadening in the NMR spectra of ANS and TNS in the presence of EpolC1576, in contrast to the sharp lines observed in the negative control experiments in the presence of dextran, suggesting interactions taking place with EpolC1576 and them being within the so-called fast exchange regime. These results encouraged us to run a preliminary exploration of the capability of the ${ }^{1} \mathrm{H}$ STD NMR technique for the first time, to the best of our knowledge, for the detection of interactions of a bacterial Epol with small molecules.

Figure 1 demonstrates that STD NMR detection of the interaction of ANS with EpolC1576 in solution is possible as strong STD signals were obtained in the difference spectrum (Figure 1A), in contrast to the absence of STD signals for a control sample containing ANS and dextran (Supplementary Figure S2). In fact, the excellent sensitivity of the resulting spectra allowed us to undertake further structural investigations to elucidate if any preferential orientation of the ligand ANS is taking place within the areas of interaction with EpolC1576. To that aim, we employed a slightly modified application of the multifrequency STD NMR methodology termed the DEEP-STD NMR approach (Monaco et al., 2017). It is important to note that we are not dealing here with a classical one-site binding type of interaction, but, instead, multiple areas of interaction are expected due to the repetitive pattern of the polysaccharide primary structure (Scheme 1). For this reason, we proposed to resort to the initial growth rate $\mathrm{STD}_{0}(\%)$ values and introduce the concept of "average orientation" in the time scale of STD NMR spectroscopy. Briefly, we carried out a comparative analysis of the binding epitope mappings of ANS obtained from a pair of STD NMR experiments using two different selective saturation conditions of EpolC1576: one at frequency $+3.22 \mathrm{ppm}$ where we directly irradiate the $\mathrm{CH}$ protons of the polysaccharide and another at $+1.22 \mathrm{ppm}$ corresponding to $\mathrm{CH}_{3}$ moiety of EpolC1576 (Figures 1B,C).

Under these conditions, significant differences in the epitope mappings were observed and the results are interpreted as follows: those protons showing the highest $\mathrm{STD}_{0}(\%)$ values (in red dots in Figures 1B,C) are oriented, on average, toward the interface of binding encoded by the particular frequency of saturation used. Using this approach, we were able to conclude that ANS interacts with a "hydrophobic" region of the EpolC1576 orienting its phenyl ring toward the $\mathrm{CH}_{3}$ group of EpolC1576 while orienting its naphthyl ring, on average, toward the $\mathrm{CH}$ moieties presumably of the rhamnose residues. These results are in excellent agreement with previous molecular simulations showing that the hydrophobic domains along EpolC1576 are associated primarily with contiguous rhamnose residues with partial O-methyl substitutions (Kuttel et al., 2017).

\section{STD NMR Study of the Interactions With Antibiotics: Ceftazidime and Kanamycin}

After the successful application of STD NMR to characterize the interactions of EpolC1576 with ANS, we decided to investigate the hypothesis that different domains on the Epol could facilitate complexation with antibiotics and slow down their diffusion through the biofilm toward the bacterial cell surface. We used ${ }^{1} \mathrm{H}$ STD NMR to characterize the interaction of the two structurally different antibiotics ceftazidime and kanamycin with EpolC1576. To this aim, we opted for two main strategies: the direct assessment of the interaction using the slightly modified DEEP-STD NMR method and an STD NMR indirect assessment by using ANS as a probe in competition assays (Wang et al., 2004). We first studied the interaction of ceftazidime with EpolC1576. As shown in Figure 2A, no signals were observed in the STD NMR experiments, which, in a first glance, would support that ceftazidime does not interact with EpolC1576.

To ensure that this result was not depending on the NMR methodology used (in fact, very slow exchange mechanisms would lead to similar STD NMR negative results), we ran a ${ }^{1} \mathrm{H}$ STD NMR competitive approach between ceftazidime and ANS. As it is shown in Figure 2B, the absence of the peaks of the ceftazidime in the STD NMR spectra and, what is more, the absence of any impact on the STD factors of ANS indicate that 

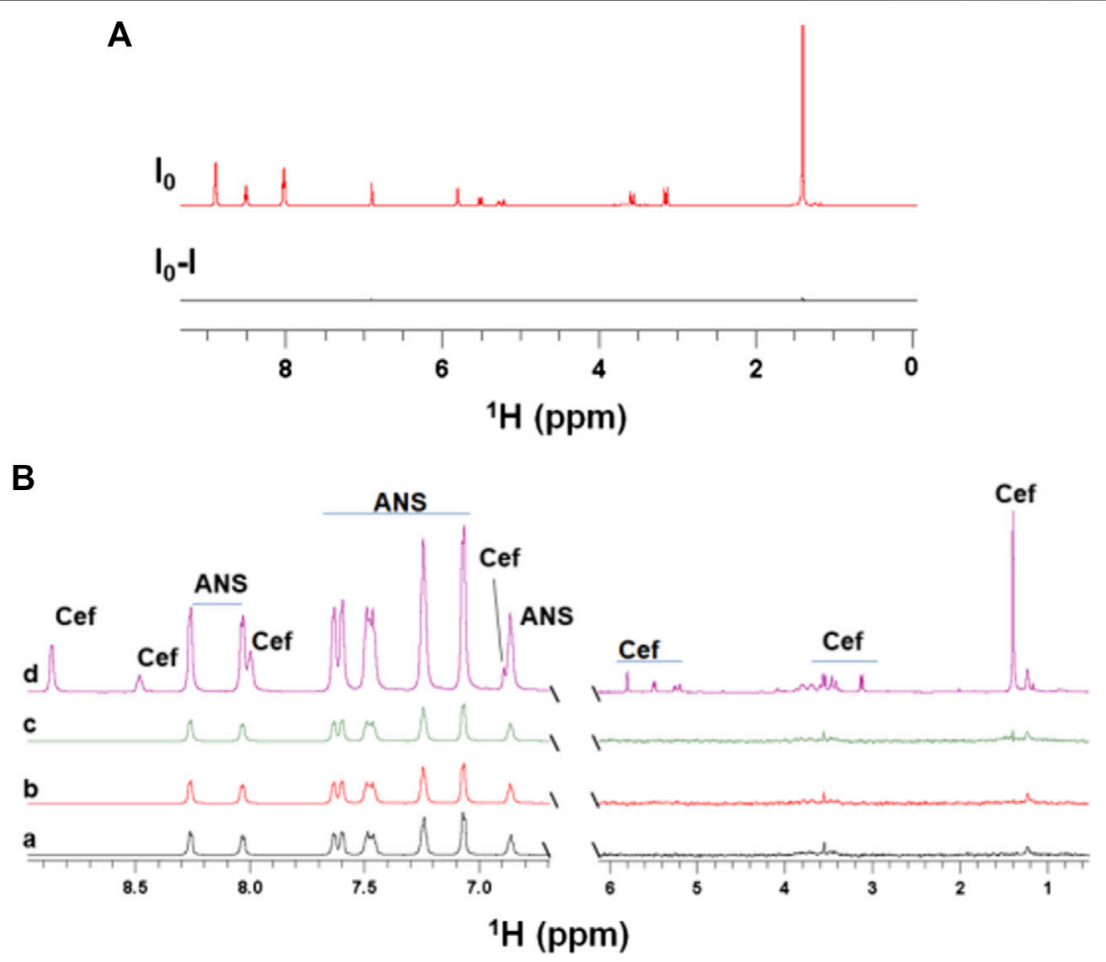

FIGURE 2 |STD NMR does not detect interactions between EpolC1576 and ceftazidime. (A) ${ }^{1} \mathrm{H}$ STD NMR spectra of ceftazidime in the presence of EpolC1576, suggesting that no interaction occurs. (B) Competition studies of ceftazidime with ANS. STD NMR spectra of ANS (5 mM) in the absence (a) and in the presence of increasing concentrations of ceftazidime in (b) $(0.5 \mathrm{mM})$, (c) $(2 \mathrm{mM})$, and (d) reference spectra.

ceftazidime is not a strong binder. Taken together, these data suggest that no binding occurs between ceftazidime and EpolC1576 in the time scale of the observation of the NMR techniques used.

However, the absence of binding of ceftazidime to EpolC1576 does not preclude an interaction that can occur at time scales not detectable by the used NMR spectroscopic techniques. For that, we decided to follow a functional approach. In fact, it is known that cephalosporines undergo degradation when in neutral or basic media so that affecting the kinetics of that degradation process can constitute a mechanism of disturbing the antibiotic efficacy (Vilanova et al., 1993). Thus, we decided to investigate whether the presence of EpolC1576 can impact the degradation rate of the antibiotic, supporting a way of EpolC1576-induced ceftazidime recalcitrance. In order to confirm this hypothesis, two samples of ceftazidime in the presence and the absence of EpolC1576 in 20 mM Tris-d11 at pH 7.5 were incubated at $298 \mathrm{~K}$.

In fact, a significant increase in the concentration of degradation products was observed in the presence of EpolC1576 after 48 and $120 \mathrm{~h}$ of incubation (Figure 3). This suggests that the EpolC1576 might contribute to the recalcitrance toward ceftazidime through degradation of the antibiotic (Figure 3). Interestingly, STD NMR experiments showed that, in contrast to ceftazidime, the degradation product interacts directly with the EpolC1576, as shown in Figure 3B. Further analysis using DOSY NMR (Figure 3C) revealed that the new species presents a set of three resonances in the aromatic region and a faster diffusion constant compared to ceftazidime. This is indicative of a molecule with smaller molecular weight. The set of NMR resonances of this low molecular weight species was confronted with the ${ }^{1} \mathrm{H}$ NMR spectra of known degradation products of ceftazidime and was assigned to pyridine (Vilanova et al., 1993).

The study was then focused on the interaction of the other antibiotic, kanamycin, with EpolC1576, following a similar strategy. In this case, the ${ }^{1} \mathrm{H}$ STD NMR experiment clearly demonstrated that kanamycin interacts with EpolC1576 in a solution (Figure 4A). Additionally, the data suggested that EpolC1576 forms a macromolecular network in water, with extremely broadened NMR signals and very efficient saturation even at $-1 \mathrm{ppm}$.

To further characterize the interaction of kanamycin with EpolC1576, a complete analysis of the orientation of the antibiotic in the area of interaction using the slightly modified DEEP-STD NMR approach was performed, as previously described (Di Micco et al., 2005; Martin et al., 2005; Souard et al., 2008; Monaco et al., 2017). Again, the interpretation of the epitope mappings obtained (Figures 4B,C) was based on the concept of "average orientation" of the protons due to the absence of a classic one-binding site interaction. As in the case of ANS, the protons showing the highest $\mathrm{STD}_{0}(\%)$ values (red dots in Figures $4 \mathrm{~B}, \mathrm{C}$ ) are oriented, on average, toward the interface of interaction with the EpolC1576. With this technique, we were also able to characterize which EpolC1576 monomers are found at the interface of interaction and are responsible for the transfer of 


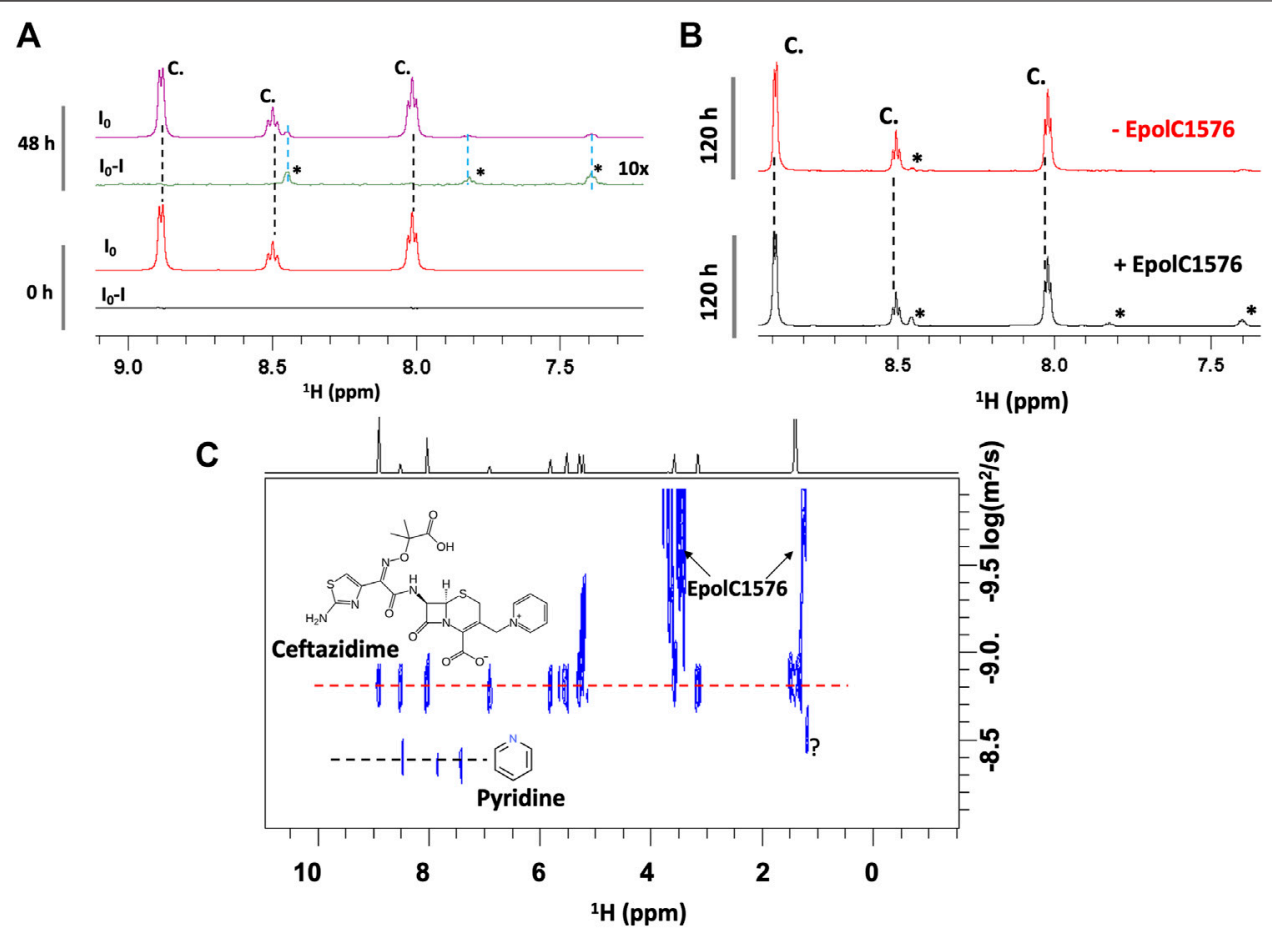

FIGURE 3|EpolC1576 accelerates the degradation of ceftazidime. (A) ${ }^{1} \mathrm{H}$ STD NMR spectra at 0 and 48 h of ceftazidime (6 mM) in the presence EpolC1576. Only the degradation product interacts with EpolC1576. (B) ${ }^{1} \mathrm{H}$ NMR spectra of ceftazidime in the presence and absence of EpolC1576 after $120 \mathrm{~h}$ in $20 \mathrm{mM}$ Tris $\mathrm{pH} 7.5$ at 298 K. The degradation product peaks are clearly visible in the presence of EpolC1576 but only slightly noticeable in its absence. (C) DOSY NMR spectra revealing the low molecular weight degradation product compared to ceftazidime. The degradation product was assigned as pyridine. The peak labeled with "?" is another degradation product yet not characterized.
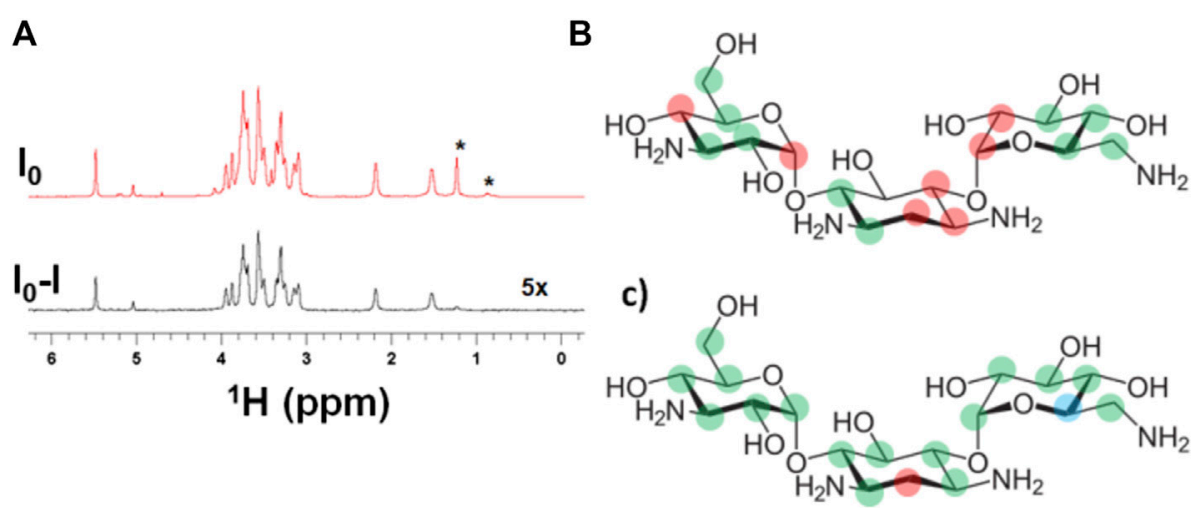

\section{= weak = intermediate = strong}

FIGURE 4 | Detection and differential binding epitope characterization of the interaction of kanamycin with EpolC1576. (A) (top) reference and (bottom) STD NMR spectra demonstrating the highly sensitive detection of the interaction of kanamycin $(2 \mathrm{mM})$ with EpolC1576 by selectively saturating EpolC1576 at -1.0 ppm, (B) binding epitope mapping of kanamycin by selectively saturating EpolC1576 at -1.0 ppm, and (C) binding epitope mapping by selectively saturating EpolC1576 at $+1.2 \mathrm{ppm}$. The resonances labeled with the asterisk (*) belong to the EpolC1576. Weak STD $_{0}(\%)=0-49 \%$, intermediate STD $(\%)=50-80 \%$, and strong STD $(\%)$ $=81-100 \%$.

saturation. Indeed, they can be derived from the saturation frequency used: $\mathrm{CH}$ and $\mathrm{CH}_{3}$ moieties at $-1 \mathrm{ppm}$ (nonselective saturation frequency) and $\mathrm{CH}_{3}$ moiety at $1.22 \mathrm{ppm}$ (selective saturation frequency). The differences between the two epitope maps obtained (Figures 4B,C) suggest that the central ring of the molecule of kanamycin is oriented, on average, toward the $\mathrm{CH}_{3}$ moiety of rhamnose monomers, in a likely similar location as the benzyl ring of ANS. 


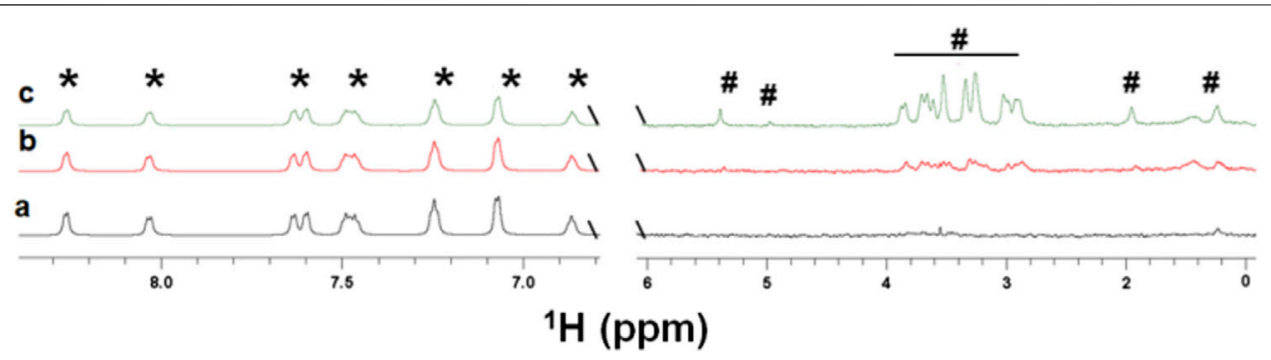

FIGURE 5 | Competition study of kanamycin with ANS. STD NMR spectra of ANS (5 mM) in the absence a) and in the presence of increasing concentrations in b $(0.5 \mathrm{mM})$ and c $(2 \mathrm{mM})$ of kanamycin. The presence of the peaks of kanamycin shows that the antibiotic is interacting with EpolC1576. However, reductions, on average, of 4 and $20 \%$ of STD factors for ANS were observed for samples in b and c (see the text) confirming its displacement. The resonances labeled with the (*) symbol belong to the ANS molecule, while those labeled with the $(")$ symbol belong to the kanamycin molecule.

We then further characterized the interaction areas of EpolC1576 with kanamycin and investigated if those areas indeed overlap with the interaction areas of ANS by performing a ${ }^{1} \mathrm{H}$ STD NMR competitive study. For this, an STD NMR spectrum at $4 \mathrm{~s}$ saturation time of a $0.5 \mathrm{mg} / \mathrm{ml}$ EpolC1576 sample containing $5 \mathrm{mM}$ ANS was acquired. Shortly after, kanamycin at different concentrations was added to this solution. The ${ }^{1} \mathrm{H}$ STD NMR spectra obtained were monitored for the presence of kanamycin peaks and for the impact on STD NMR factors of ANS.

The spectra (Figure 5 b,c) show that kanamycin binds to the EpolC1576 in the presence of ANS. Furthermore, an increase in the concentration of kanamycin (Figure $5 \mathrm{~b}, \mathrm{c}$ ) led to a progressive reduction in STD factors of ANS ( 4 and 20\% reduction for 0.5 and $2 \mathrm{mM}$ of kanamycin, respectively). Taken together, these data strongly support that kanamycin displaces ANS from its interaction sites and that their binding areas overlap, confirming the interpretation of the multifrequency STD NMR results with kanamycin described above.

\section{CONCLUSION}

Here, we have demonstrated that multifrequency STD NMR experiments are extremely sensitive spectroscopic tools to: 1) confirm binding of small molecules to bacterial biofilm-forming exopolysaccharides, as that produced by $B$. multivorans (EpolC1576), and 2) provide structural details at an atomic level of the interactions as well as average information about the orientation and area of interaction of the small molecule within the different regions of the exopolysaccharide.

It is well known that bacterial recalcitrance is mediated through several mechanisms. In this study, we have investigated the contribution and extent of involvement in recalcitrance of the exopolysaccharide EpolC1576 produced by $B$. multivorans. Confronting the data between the two antibiotics, two general but very different mechanisms can be proposed as models to explain EpolC1576 contribution to recalcitrance. The first one, regarding kanamycin, suggests that EpolC1576 might contribute to recalcitrance toward this antibiotic by direct interaction with it. The second one, regarding ceftazidime, suggests another type of mechanism involving an increase in the ceftazidime degradation rate where the role of EpolC1576 seems to be fundamental.

This proof of principle of the applicability of multifrequency STD NMR to interactions of small molecules with biofilm exopolysaccharides is of high relevance to studies to come about the structural details behind the impact of interactions of biofilm forming exopolysaccharides with small molecules, such as nutrients or drugs, on bacterial recalcitrance, where STD NMR approaches open a door to access atomic detail information on the binding modes and areas of interactions of the ligands.

\section{DATA AVAILABILITY STATEMENT}

The raw data supporting the conclusion of this article will be made available by the authors, without undue reservation.

\section{AUTHOR CONTRIBUTIONS}

RN: methodology, data production, data analysis, and writing-original draft, review, and editing. SM: data production, data analysis, and writing-review and editing. MD: polysaccharide production, data analysis, and writing-review and editing. RR: supervision, funding acquisition, and writing - review and editing. PC: supervision, funding acquisition, and writing-review and editing. JA: supervision, conceptualization, funding acquisition, methodology, and writing-review and editing.

\section{FUNDING}

This work was supported by the Spanish Ministry of Science, Innovation and Universities through the PID 2019109395GB-I00 project and the Biotechnology and biological Sciences Research Council (BBSRC) New Investigator grant (BB/P010660/1), both awarded to JA. Similarly, RN and SM were supported by BB/P010660/1. JA also acknowledges financial support from the Universidad de Sevilla (Acciones 
Especiales del VI Plan Propio de Investigación y Transferencia). This publication was supported by an agreement with Cornell University, Food Science (S), under Prime Agreement 1R01GM123283-01 from NATL INST OF HEALTH DHHS.

\section{REFERENCES}

Airoldi, C., Giovannardi, S., La Ferla, B., Jiménez-Barbero, J., and Nicotra, F. (2011). Saturation Transfer Difference NMR Experiments of Membrane Proteins in Living Cells under HR-MAS Conditions: the Interaction of the SGLT1 Co-transporter with its Ligands. Chem. Eur. J. 17, 13395-13399. doi:10.1002/chem.201102181

Angulo, J., Enríquez-Navas, P. M., and Nieto, P. M. (2010). Ligand-receptor Binding Affinities from Saturation Transfer Difference (STD) NMR Spectroscopy: the Binding Isotherm of STD Initial Growth Rates. Chem. Eur. J. 16, 7803-7812. doi:10.1002/chem.200903528

Benie, A. J., Moser, R., Bäuml, E., Blaas, D., and Peters, T. (2003). Virus-Ligand Interactions: Identification and Characterization of Ligand Binding by NMR Spectroscopy. J. Am. Chem. Soc. 125, 14-15. doi:10.1021/ ja027691e

Calabrese, V., Muñoz-García, J. C., Schmitt, J., da Silva, M. A., Scott, J. L., Angulo, J., et al. (2019). Understanding Heat Driven Gelation of Anionic Cellulose Nanofibrils: Combining Saturation Transfer Difference (STD) NMR, Small Angle X-ray Scattering (SAXS) and Rheology. J. Colloid Interf. Sci. 535, 205-213. doi:10.1016/j.jcis.2018.09.085

Di Micco, S., Bassarello, C., Bifulco, G., Riccio, R., and Gomez-Paloma, L. (2005). Differential-frequency Saturation Transfer Difference NMR Spectroscopy Allows the Detection of Different Ligand-DNA Binding Modes. Angew. Chem. Int. Ed. Engl. 45, 224-228. doi:10.1002/anie.200501344

Dolfi, S., Sveronis, A., Silipo, A., Rizzo, R., and Cescutti, P. (2015). A Novel Rhamno-Mannan Exopolysaccharide Isolated from Biofilms of Burkholderia Multivorans C1576. Carbohydr. Res. 411, 42-48. doi:10.1016/ j.carres.2015.04.012

Flemming, H.-C., Wingender, J., Szewzyk, U., Steinberg, P., Rice, S. A., and Kjelleberg, S. (2016). Biofilms: an Emergent Form of Bacterial Life. Nat. Rev. Microbiol. 14, 563-575. doi:10.1038/nrmicro.2016.94

Flemming, H. C. (2016). EPS-then and Now. Microorganisms 4 (4), 41. doi:10.3390/microorganisms4040041

Hall, C. W., and Mah, T.-F. (2017). Molecular Mechanisms of Biofilm-Based Antibiotic Resistance and Tolerance in Pathogenic Bacteria. FEMS Microbiol. Rev. 41, 276-301. doi:10.1093/femsre/fux010

Kuttel, M. M., Cescutti, P., Distefano, M., and Rizzo, R. (2017). Fluorescence and NMR Spectroscopy Together with Molecular Simulations Reveal Amphiphilic Characteristics of a Burkholderia Biofilm Exopolysaccharide. J. Biol. Chem. 292, 11034-11042. doi:10.1074/jbc.m117.785048

Limoli, D. H., Jones, C. J., and Wozniak, D. J. (2015). Bacterial Extracellular Polysaccharides in Biofilm Formation and Function. Microbiol. Spectr. 3 (3), MB-0011-2014. doi:10.1128/microbiolspec.mb-0011-2014

Martin, J. N., Muñoz, E. M., Schwergold, C., Souard, F., Asensio, J. L., JiménezBarbero, J., et al. (2005). Carbohydrate-Based DNA Ligands: Sugar-Oligoamides as a Tool to Study Carbohydrate-Nucleic Acid Interactions. J. Am. Chem. Soc. 127, 9518-9533. doi:10.1021/ja050794n

\section{SUPPLEMENTARY MATERIAL}

The Supplementary Material for this article can be found online at: https://www.frontiersin.org/articles/10.3389/fmolb.2021.727980/ full\#supplementary-material

Mayer, M., and James, T. L. (2004). NMR-based Characterization of Phenothiazines as a RNA Binding Scaffold †. J. Am. Chem. Soc. 126, 4453-4460. doi:10.1021/ja0398870

Mayer, M., and Meyer, B. (1999). Characterization of Ligand Binding by Saturation Transfer Difference NMR Spectroscopy. Angew. Chem. Int. Ed. 38, 1784-1788. doi:10.1002/(sici)1521-3773(19990614)38:12<1784::aid-anie1784>3.0.co;2-q

Monaco, S., Tailford, L. E., Juge, N., and Angulo, J. (2017). Differential Epitope Mapping by STD NMR Spectroscopy to Reveal the Nature of Protein-Ligand Contacts. Angew. Chem. Int. Ed. 56, 15289-15293. doi:10.1002/anie.201707682 Scoffone, V. C., Chiarelli, L. R., Trespidi, G., Mentasti, M., Riccardi, G., and Buroni, S. (2017). Burkholderia Cenocepacia Infections in Cystic Fibrosis Patients: Drug Resistance and Therapeutic Approaches. Front. Microbiol. 8, 1592. doi:10.3389/fmicb.2017.01592

Souard, F., Muñoz, E., Peñalver, P., Badía, C., del Villar-Guerra, R., Asensio, J. L., et al. (2008). Sugar-oligoamides: Bound-State Conformation and DNA MinorGroove-Binding Description by TR-NOESY and Differential-Frequency Saturation-Transfer-Difference Experiments. Chem. Eur. J. 14, 2435-2442. doi:10.1002/chem.200701103

Vilanova, B., MuñozBlanco, F. F. G., Donoso, J., and Blanco, F. G. (1993). HPLC and1H-NMR Studies of Alkaline Hydrolysis of Some 7-(Oxyiminoacyl) cephalosporins. Hca 76, 2789-2802. doi:10.1002/hlca.19930760805

Wang, Y.-S., Liu, D., and Wyss, D. F. (2004). Competition STD NMR for the Detection of High-Affinity Ligands and NMR-Based Screening. Magn. Reson. Chem. 42, 485-489. doi:10.1002/mrc.1381

Worthington, R. J., Richards, J. J., and Melander, C. (2012). Small Molecule Control of Bacterial Biofilms. Org. Biomol. Chem. 10, 7457-7474. doi:10.1039/ c2ob25835h

Wu, D. H., Jhonson, A. D., and Chen, C. S. (1995). An Improved DiffusionOrdered Spectroscopy experiment Incorporating Bipolar-Gradient Pulses. J. Magn. Reson. Ser. A 115, 260-264. doi:10.1006/jmra.1995.1176

Conflict of Interest: The authors declare that the research was conducted in the absence of any commercial or financial relationships that could be construed as a potential conflict of interest.

Publisher's Note: All claims expressed in this article are solely those of the authors and do not necessarily represent those of their affiliated organizations, or those of the publisher, the editors, and the reviewers. Any product that may be evaluated in this article, or claim that may be made by its manufacturer, is not guaranteed or endorsed by the publisher.

Copyright (c) 2021 Nepravishta, Monaco, Distefano, Rizzo, Cescutti and Angulo. This is an open-access article distributed under the terms of the Creative Commons Attribution License (CC BY). The use, distribution or reproduction in other forums is permitted, provided the original author(s) and the copyright owner(s) are credited and that the original publication in this journal is cited, in accordance with accepted academic practice. No use, distribution or reproduction is permitted which does not comply with these terms. 